\begin{tabular}{l} 
SCIENCE \& TECHNOLOGY \\
Journal homepage: http://www.pertanika.upm.edu.my/ \\
\hline PERTANIKA
\end{tabular}

\title{
LTE Network Analysis in Frequency Reuse Recycling Techniques
}

\author{
Muhammad Sabir Hussain*, Nasri Suleiman and Nor Kamariah Noordin \\ Department of Electrical and Electronics Engineering, Faculty of Engineering, University Putra Malaysia, \\ 43400 UPM, Serdang, Selangor, Malaysia
}

\begin{abstract}
In recent years, several researchers have embraced fractional frequency (FF) reuse as a strategy for resolving the inter-cell and co-channel interferences of adjacent cells (ICI, $\mathrm{CCI}$ ) as the number of wireless networks grows. This technique is focused on the cell division of two parts, the inner and the outer, which enables multiple frequency bands to be assigned. The frequency advantages can be completely used in each inner zone, since there is no inter-cell disturbance for consumers in inner regions. According to this effective usage of the frequency spectrum available, FF will reduce the interruption of the channel and improve device efficiency. This manuscript presents a comprehensive study of different mechanisms to select the optimal FF scheme based on the user throughput. The analysis was conducted in order to obtain the optimal internal and external range for the cells as well as the optimal frequency distribution between the areas of the FR, Fractional Frequency Reuse 1 (FFR1) and Fractional Frequency Reuse 2 (FFR2) and evaluating their outputs and their number of users. In detail the overall consumer efficiency through the configured frequency distribution is analyzed. The FFR is a resource allocation technique that can effectively mitigate inter-cell interference (ICI) in LTE based HetNets and it is a promising solution. The proposal also employs high number sectors in a cell, low interference and good frequency reuse. The processes are tested by way of multiple modeling simulations.
\end{abstract}

ARTICLE INFO

Article history:

Received: 23 June 2020

Accepted:25 November 2020

Published: 22 January 2021

DOI: https://doi.org/10.47836/pjst.29.1.27

E-mail addresses:

engineersabir1@yahoo.com (Muhammad Sabir Hussain)

nasrisulaiman@upm.edu.my (Nasri Suleiman)

nknordin@upm.edu.my (Nor Kamariah Noordin)

* Corresponding author
Keywords: FFR, inner region, LTE network, maximum throughput, optimal radius, outer region

\section{INTRODUCTION}

The 3rd Generation Long Term Innovation Collaboration Project (3GPP-LTE) is the 4th Generation (4 G), a wireless network built to serve compact, powerful and highperformances end-users, providing a large 
capacity, improved connectivity efficiency. In December 2007 3GPP published their first LTE research work in "release 7," which now has a major topic (Hashima et al., 2014; Simonsson, 2007). LTE Multi-Access Orthogonal Frequency Division (OFDMA) technology for downlinks has attracted significant attention from researchers in recent years in achieving a high rate of data transfer in wireless and mobile communications. OFDMA provides a higher spectrum output in order to satisfy the growing demands of smartphone users because of its versatile frequency assignment (Zhang et al., 2020). In LTE, each OFDMA traffic channel is solely allocated to one individual, which provides the end-user frequency with a high degree of reliability and flexibility (Zhao et al., 2012). The device efficiency is significantly degraded by the Inter-cell Interference (ICI) dependent on a multi cell LTE OFDMA-related network because of the same frequency reuse and interference by the co-channel (CCI) when more than one radio transmitters use the same frequency (Chung et al., 2014; Elayoubi et al., 2008; Hindia et al., 2014; Mannweiler et al., 2020; Wang et al., 2014).

In order to address both ICI and CCI, FFR has become an area of intensive study by several OFDMA-based researchers (Bilios et al., 2013; Mao et al., 2008; Nuaymi, 2007). In order to minimize the CCI and ICI, FFR main target is to divide cells volume into inner and outer regions (Chang et al., 2009; Cong et al., 2020; Group, 2004; Saleh, 2020). FFR is a mixture of different types of frequency reuse scheme such as traditional frequency reuse (FR) factor and FFR3. One of the main objectives of LTE is to use the whole of the system's bandwidth to achieve high spectral efficiency (Group, 2004; Mao et al., 2008). This method is known as traditional FR where all available bandwidth is fully assigned in each cell. In FRR3, on the other hand, the available bandwidth is divided into 3 equal sub-bands and each of these sub-bands will be allocated in a manner to the cells in a cluster so that no adjacent cells will have the same sub-bands. In addition, traditional FR can be used by the mobile stations (MSs) which are located around the base stations (BSs) or in particular in the cell inner region and FRR3 is allocated to the cell edge users, which will decrease the interference, but data rate also will be decreased due to the fact that the full frequency band is not used by this method (Ali \& Leung, 2009; Chang et al., 2009; Kim et al., 2014; Soultan et al., 2020; Stiakogiannakis \& Kaklamani, 2010). OFDMA allows the dynamic allocation of subcarriers (called OFDMA traffic channel) to different users at different time instances. For example, it utilizes $15 \mathrm{KHz}$ subcarriers in LTE, which can be grouped into Resource Blocks (RBs) with each having 12 subcarriers (Kim et al., 2010; Lei et al., 2007; Sheu et al., 2015; Taranetz et al., 2011; Wang, 2013). To implement these for FFR scheme, one of the ways to allocate these RBs is to allocate them in a sector (Guo \& Suárez, 2020; Hambebo et al., 2014). The main goal of this paper is to investigate the characteristics of three existing methods of FR, FFR1 and FFR2 to evaluate an interference management in LTE networks. The studied mechanism calculates the optimal FFR scheme based on user throughput. It then successively checks the inner cell radius and the inner 
cell frequency and calculates the Signal to Interference Plus Noise Ratio (SINR), capacity and throughput. These values are then used in order to calculate the cell mean throughput. Finally, the mechanism selects the optimal center to cell radius ratio and optimal number of users in order to propose the best FFR scheme that maximizes the cell mean throughput (Rumney, 2013). The rest of the paper is organized as follows: Section 2 describes the related work to our study. Section 3 refers to our studied system model, Section 4 introduces the simulation, and Section 5 presents the simulation results and discussion.

\section{RELATED WORK}

The research on FFR focuses on the implementation of reuse of frequencies, which is the sub band divisioning for the BS area. OFDMA infrastructure has been used by several wireless network protocols, like GPP and 3GPP2. In recent study, the key topic of discussion is network efficiency, performance, rate, scope, and frequency distribution. Hindia et al. (2014) selected the frequency reuse pattern from four varying degrees of partitioning criteria (e.g. FRF1 and FRF3) to improve the performance of mobile and base station. FFR impact was presented in the cellular area division into inner and outer regions was suggested to avoid conflict in Bilioss et al. (2013) as an effect of FFR. The authors proposed partial frequency allocation for the cell edge region and full frequency spectrum for the cell inner region. The users are modulated more tightly than BSs, because different modulation instructions are taken into consideration according to the distance of the consumer from BSs and also the state of the signal, which indicates that the frequency reuse is higher. In most research, static or semi-static synchronization systems are introduced in BSs with little cellular performance benefit and needing advance frequency preparation. The system's efficiency is significantly diminished to increase the cell-end performance (Mao et al., 2008; Nuaymi, 2007). In contrast, prior frequency planning is not required in dynamic coordination technique. FFR was proposed in order to keep the stability between celledge throughput and overall network throughput (Chang et al., 2009; Group, 2004). In these works the authors partitioned cells into inner and edge zones which associated the low and high Frequency Reuse Factors In order to reduce interference, the total available frequency bandwidth was divided into two sub-bands such as center band and edge band where frequency bands are fully to Bfr and partly to Bpr allocated respectively. Ali and Leung (2009) implemented the super-group and the hierarchical organization of a standard community, which were divided in cells and sectors to ensure a greater device efficiency. The FFR efficiency improvement was analyzed by Stiakogiannakis and Kaklamani (2010) in three forms of zone distributions, such as SINR-based, distance-based and load-balancebased techniques. The combination of the load balancing with the SINR and the correct distribution of resources gives the machine a decent bit rate. Business technology had been suggested by Kim et al. (2014) for the frequency reuse component. This method is used in WiMAX to boost the $802.16 \mathrm{j}$ relay device that transmits the signals to the BS portfolio. 
Based on the simulation test, this scheme improved the spectrum utilization and network output. Macro-femic networks with the idea of FFR, there is a novel hierarchical resource distribution method suggested by Lei et al. (2007). The three considerations in this scheme to minimize the CCI in macro and femtocells are size, time and place. To this end the method suggested a hybrid two-part grouping of macro cells and the deployment of femto BSs in each section of macro cells conducted in accordance with the objective of growing the average cell efficiency. Improved FFR technique (E-FFR) was introduced in WiMAX by Kim et al. (2010) for reducing ICI. The variety of users and frequency are viewed as strategies to boost device efficiency for mobile and fixed users. Such strategies improve the signal from the consumer against undesired noise. Downlink (DL) and Uplink (UL) frames were divided into WiMAX frames, and each frame was further divided into zones to quantify unused space to allow appropriate modulation and coding in order to improve device efficiency. However, overhead will occur at BS without continuous consumer input as the channel parameters alter over time. A new multiple access (B-OFDMA) biorthogonal frequency division system with the broad low-complexity Fourier transform fraction (FrFT) was proposed by Wang (2013). The variation of time and frequency is set at each BS in this scheme with multi-angle reuse (MADR). The program will efficiently reduce ICI and increase the efficiency of the device. A FFR optimization method based on capacity density was suggested by Taranetz et al. (2011). In this work, the frequency sub-band with highest achievable capacity density was assigned to a given user. The authors then formulated the FFR optimization problem mathematical expressions. Here, the per-user capacity was mainly investigated from per-area capacity. The maximum average capacity density was aimed with the condition of least required cell-edge throughput $\mathrm{c} 5 \%$ and minimizing the c95\% peak user throughput loss.

\section{METHOD AND SYSTEM MODEL}

In this section, SINR and capacity of user $\mathrm{x}$ on subcarrier $\mathrm{n}$ are calculated based on theoretical approach in order to define the throughput. The $N$ adjacent cells are considered as overall network where a number of users look forward to share a collection of subcarriers in each cell. In this case a distinction of a user which can be found in the inner or outer zone of the cell. In an OFDMA based network, we assume the user as $\mathrm{x}$ in a base station $s$ on subcarrier $c$, the related SNIR can be calculated using Equation 1 (Chung et al., 2014; Hindia et al. 2014; Wang et al. 2014):

$$
\operatorname{SINR}_{x, n}=\frac{L_{s, x} \cdot P_{S, c} \cdot G_{s, x, c} \cdot}{\sigma_{c}^{2}+\sum_{j=1}^{k} L_{j, x} \cdot P_{j, c} \cdot G_{j, x, c}}
$$

where the path loss associated with channel between user $\mathrm{x}$ and base station $\mathrm{s}$ is defined as to $\mathrm{L}_{\mathrm{s}, \mathrm{x}}$, the transmit power of the base station as $\mathrm{P}_{\mathrm{s}, \mathrm{c}}$, exponentially distributed channel fast 
fading power as $\mathrm{G}_{\mathrm{s}, \mathrm{x}, \mathrm{c}}$, the noise power of the additive White Gaussian Noise (AWGN) channel is defined as $\sigma c^{2}$. The sets of all interfering base stations are defined as symbol $\mathrm{k}$ and $\mathrm{j}$. More precisely, $k$ is the number of co-channel cell and $j$ is the cell index. We consider the transmit power on subcarrier is equal as $P_{s, c}=P$ for all BSs. The coefficient $G_{s, x, c}$ is equal to 1 of its mean value.

The interference presents in the inner and the outer regions of the disjoint set of download links. A specific frequency band is assigned on transmission, which is a common band in every inner region, thus it brings interference in those regions. Moreover, it is required to distinguish two different types of BSs. The first type of consists of all interfering BSs, which transmits to the users of the cell-inner region on the same sub-band as user $\mathrm{x}$ and the second type consists of all interfering BSs transmits to the users of the cell-edge users on the same sub-band as user $\mathrm{x}$. Now, in order to calculate the throughput, we first define the capacity of the user. The capacity of user $\mathrm{x}$ on subcarrier $\mathrm{c}$ is given by the following Equation 2 (Bilios et al., 2013):

$$
E_{x, c}=\Delta f \cdot \log _{2}\left(1+S I N R_{x, c}\right)
$$

Where, $\Delta f$ denotes as the available bandwidth for each subcarrier. Here, the subcarrier is divided among the number of users to be shared. Finally, the throughput of the user $x$ is expressed specifically in terms of SINR and $E_{\mathrm{x}, \mathrm{c}}$ as in Equation 3

$$
T_{x}=\sum_{n} \beta_{x, n} \cdot E_{x, n}
$$

Where $\beta_{x, c}$ refers to the subcarrier assigned to user $x$. When, $\beta_{\mathrm{x}, \mathrm{c}}=1$, the subcarrier $\mathrm{c}$ is allocated to user $x$. Otherwise, $\beta_{\mathrm{x}, \mathrm{c}}=0$.

\section{SIMULATION ENVIROMENT}

The applications are tested and evaluated using the Matlab program. As detailed in Table 1 , the simulation area consists of 7 cells network and the users randomly distributed in the cells and system bandwidth is $10 \mathrm{MHZ}$.

Figure 1 is the graphical representation of the network area for FFR1 scheme where it is being applied to investigate the optimum center ratio and the optimum number of users per cell. As we can observe from the Figure 1 how the users are distributed throughout the inner and outer cells where we assume the area of $X$-axis from $-4000 \mathrm{~m}$ to $3000 \mathrm{~m}$ and Y-axis from $-3000 \mathrm{~m}$ to $4000 \mathrm{~m}$. The inner and outer cell have specific sub-band which is represented by band color. The different colors in Figure 1 correspond to bandwidth of the different cells. FFR1 is a heavy frequency and low frequency reuse hybrid. Strong frequency 
reuse splits the bandwidth into different subbands(color) depending on the reuse factor selected such that the neighboring cells will communicate on different subbands(color). The entire bandwidth of FFR1 is split into internal and external subbands. The central area is allocated to local users residing outside BS on the basis of path failure with a decreased power that relates to the FRF1 of one that is fully utilized by all eNBs. The fraction of the outer bandwidth area is distributed for more than one frequency reuse factor. Both BSs are required to share the entire bandwidth of the soft frequency. However, the eNBs are restricted to a certain capacity constraint for increasing subcontractor transmission. In fact, the number of cell users is assigned arbitrarily.

Figure 2 is the demonstration of the total FFR2 network region to analyze the optimal center ratio and the optimal consumer number per node. The distribution of users in the internal and external cells of the cluster in the form of sub-bands (color) is shown in Figure

Table 1

Simulations inputs

\begin{tabular}{cc}
\hline Parameters & Settings \\
\hline Cell Radius & $750 \mathrm{~m}$ \\
Bandwidth & $10 \mathrm{MHz}$ \\
Number of Cell & 7 \\
Number of Subcarriers & 50 \\
Bearer Types & From QCI 1- QCI 15 \\
eNodeB Height & $50 \mathrm{~m}$ \\
UE Height & $1 \mathrm{~m}$ \\
eNodeB Tx power in cell Center & $40 \mathrm{dBm}$ \\
eNodeB Tx power in cell edge & $46 \mathrm{dBm}$ \\
Path loss & Cost $231 \mathrm{Hata} \mathrm{Model} \mathrm{(dB)}$ \\
Power Noise Density & $-174 \mathrm{dBm} / \mathrm{Hz}$ \\
\hline
\end{tabular}

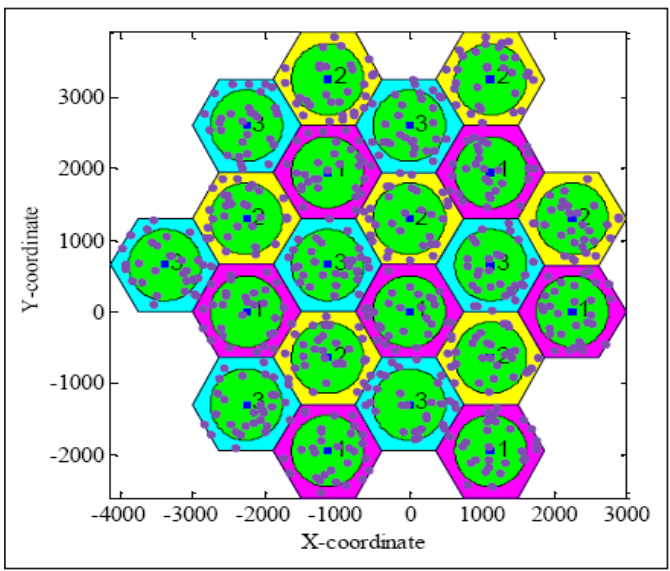

Figure 1. Network area of FFRI

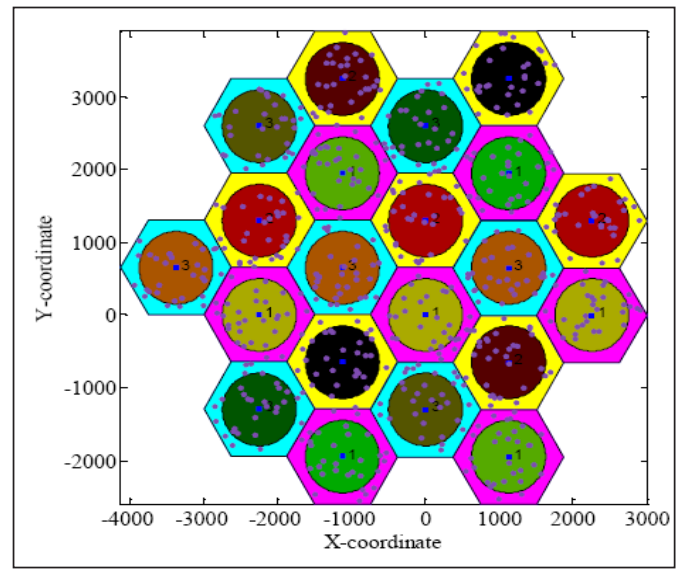

Figure 2. Network area of FFR2 
2. The $\mathrm{X}$-axis field from -4000 meters to 3000 meters and the $\mathrm{Y}$-axis from -3000 meters to 4000 meters as for FFR1 are expected to be comparable. The frequency reuse component is greater than one for the proportion of the main and main regions of bandwidth. Except for inner and outer parts, the frequency bands vary.

\section{RESULTS AND DISCUSSION}

Figure 3 and Table 2 represent the average cell throughput according to the center radius to cell radius ratio with different number of users for FFR1. We can observe from the Figure 3 that the throughput is almost linearly increasing until cell radius ratio 0.5 for all groups of users except group of 10 users. However, the slope of the line graph varies based on the number of users. The average throughput of a group of 10, 20, 30, 40, 50, 60, 70, and 80

Table 2

The maximum throughput (Mbps) for the various cell center ratio and users for FFR1

\begin{tabular}{cccccccccc}
\hline User & Radius & $\mathbf{0 . 3}$ & $\mathbf{0 . 4}$ & $\mathbf{0 . 5}$ & $\mathbf{0 . 6}$ & $\mathbf{0 . 7}$ & $\mathbf{0 . 8}$ & $\mathbf{0 . 9}$ \\
\hline & $\mathbf{1 0}$ & 13 & 12.8 & 12.5 & 12 & 11.6 & 10.8 & $\mathbf{1 0 . 5}$ \\
& $\mathbf{2 0}$ & 16 & 17.5 & 20.3 & 22 & 22.5 & 21.5 & $\mathbf{2 1}$ \\
& $\mathbf{3 0}$ & 19 & 22 & 26 & 29.8 & 32.5 & 31.5 & $\mathbf{2 7 . 5}$ \\
& $\mathbf{4 0}$ & 21 & 25.5 & 31.5 & 36.5 & 39 & 34 & $\mathbf{2 7 . 5}$ \\
& $\mathbf{5 0}$ & 23 & 29.8 & 36 & 42 & 41.5 & 35 & $\mathbf{2 7 . 5}$ \\
$\mathbf{6 0}$ & 26 & 33 & 41.5 & 45 & 41.5 & 36.5 & $\mathbf{2 7 . 5}$ \\
& $\mathbf{7 0}$ & 28 & 37.5 & 46 & 45.5 & 41.5 & 36.6 & $\mathbf{2 7 . 5}$ \\
& $\mathbf{8 0}$ & 31 & 41 & 48 & 46 & 41.5 & 37 & $\mathbf{2 7 . 5}$ \\
\hline
\end{tabular}

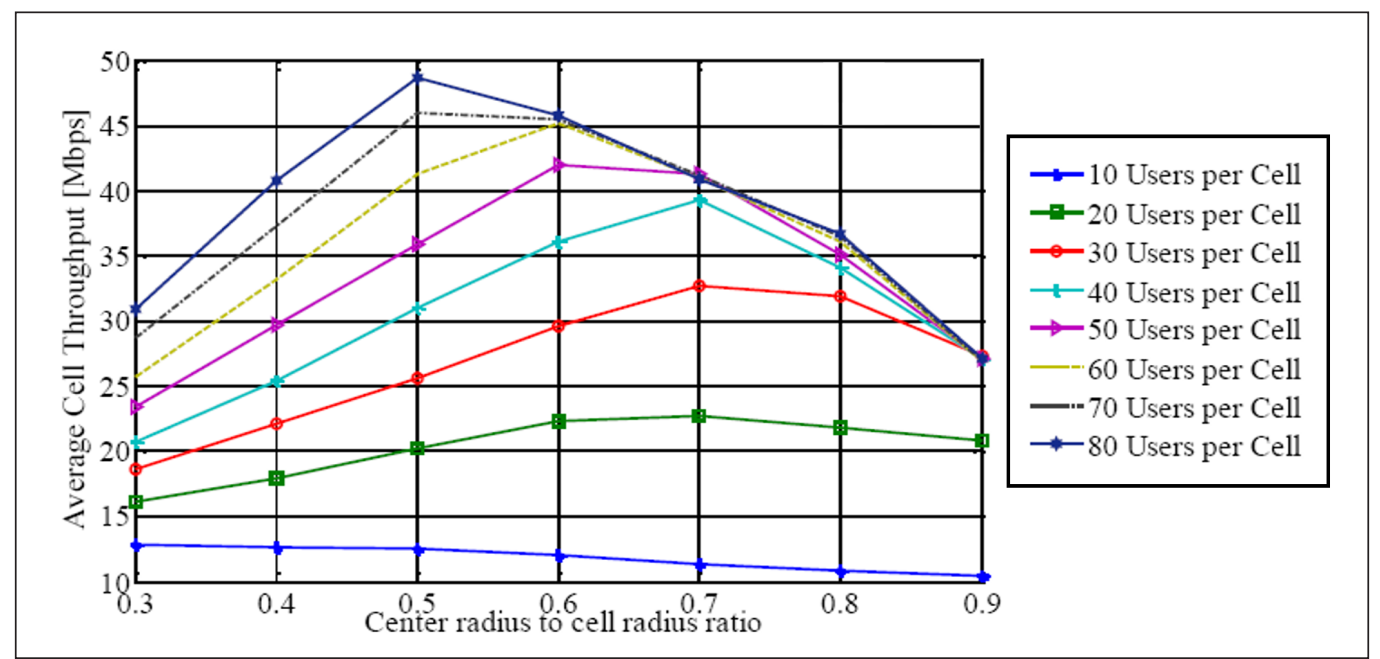

Figure 3. The average cell throughput according to the variation of cell radius to cell center ration with various user group for FFR1. 
users are $12.5,20.5,26,31.5,36,41.5,46,48 \mathrm{Mbps}$, respectively when the center to cell radius ratio $\left(\mathrm{C}_{\mathrm{rr}}\right)$ is 0.5 . Remarkably, the average throughput is highest for group of 80 and 70 users when the $C_{\mathrm{rr}}$ is 0.5 and the breakdown points for group of 60 and 50 users are at $\mathrm{C}_{\mathrm{rr}} 0.6$ and 40,30, 20 users are at 0.7. After those points the throughput linearly decreases with the increase of the cell radius ratio. From the above scenario, this can be concluded that the highest average throughput observed when the $C_{r r}$ is $50 \%$ for the group of 80 users.

The average cell throughput according to the number of users per cell with the various cell center radius ratio is being observed in Figure 4 more intuitively. The throughput linearly increases for the $30 \%, 40 \%, 50 \%$ and $60 \%$ of center radius ratio, respectively with the increase number of users. However, the throughput becomes saturated for the $\mathrm{C}_{\mathrm{rr}} 70 \%$, $80 \%$ and $90 \%$ after the certain number of users. The throughput is seen highest when the number of users are as higher as 80 at the $\mathrm{C}_{\mathrm{rr}} 50 \%$.

The average performance in Figure 5 and Table 3 is seen in terms of the number of FFR2 users with the center radius ratio. The linear rise in performance for the 80, 70, 60, 50 and 40 users until the specified center-ratio is shown in this chart. On the other side, the averages are almost the same for the community of 30 and 40 users, while the performance for the category of 10 users is often smaller. Like FFR1, a limit of $60 \mathrm{Mbps}$ for the 80 users at Crr 0.6 is available. Figures 3 and 5 demonstrate that the average FF2 efficiency is higher than FFR2 and the ideal cell center radius is $60 \%$.

Figure 6 shows the representation of the average user output by the cell-to-center ratio. For the group of ten to twenty users, for all variations of center radius the variation of the throughput values are almost the same. With the center radius ratio from $30 \%$ to $70 \%$, the output increases linearly with various slopes where $80 \%$ and $90 \%$ of the output for a given

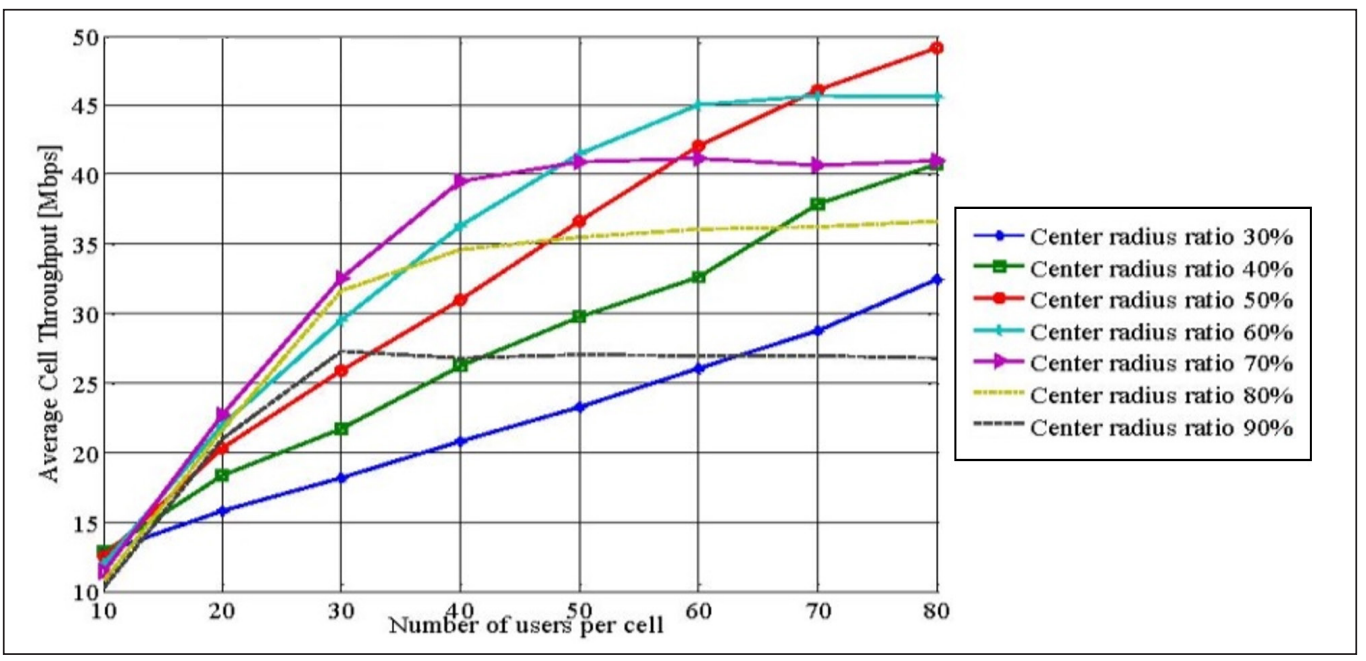

Figure 4. The average cell throughput at cell radius $750 \mathrm{~m}$ according to the number of users per cell to center radius ratio 


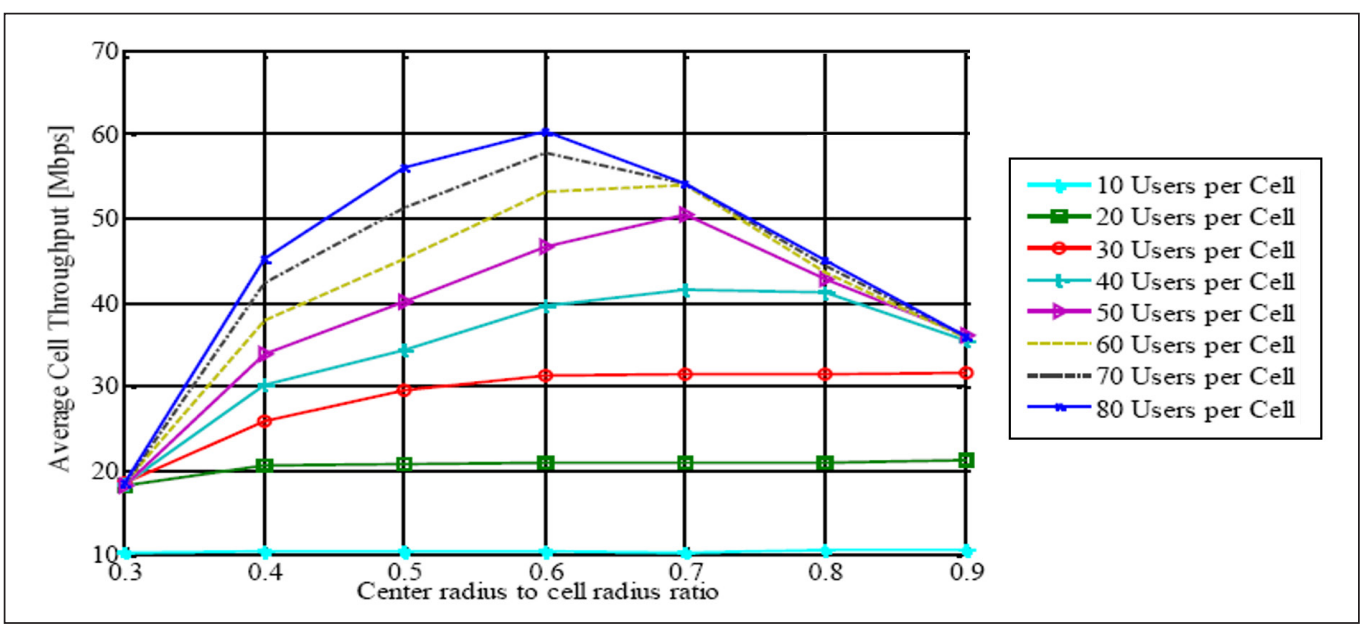

Figure 5. Average cell throughput at cell radius $750 \mathrm{~m}$ according to the variation of cell radius to cell center ration with users for FFR2.

Table 3

The maximum throughput (Mbps) for the various cell center ratio $\left(C_{r r}\right)$ and users for FFR2

\begin{tabular}{ccccccccc}
\hline User & Radius & $\mathbf{0 . 3}$ & $\mathbf{0 . 4}$ & $\mathbf{0 . 5}$ & $\mathbf{0 . 6}$ & $\mathbf{0 . 7}$ & $\mathbf{0 . 8}$ & $\mathbf{0 . 9}$ \\
\hline & $\mathbf{1 0}$ & 10 & 10.1 & 10.1 & 10.1 & 10.1 & 111 & $\mathbf{1 1}$ \\
& $\mathbf{2 0}$ & 19 & 20.5 & 20.5 & 20.5 & 20.5 & 20.5 & $\mathbf{2 1}$ \\
& $\mathbf{3 0}$ & 19 & 25 & 29 & 31.5 & 31.6 & 31.8 & $\mathbf{3 2}$ \\
$\mathbf{4 0}$ & 19 & 30 & 34 & 40 & 42 & 41.6 & $\mathbf{3 6}$ \\
& $\mathbf{5 0}$ & 19 & 34 & 40 & 46.5 & 50 & 43 & $\mathbf{3 6 . 5}$ \\
$\mathbf{6 0}$ & 19 & 38 & 45 & 54 & 54.5 & 43.2 & $\mathbf{3 6 . 5}$ \\
& $\mathbf{7 0}$ & 19 & 42 & 52 & 57.6 & 54.5 & 43.4 & $\mathbf{3 6 . 5}$ \\
$\mathbf{8 0}$ & 19 & 45 & 56 & 60 & 54.5 & 44 & $\mathbf{3 6 . 5}$ \\
\hline
\end{tabular}

number of users is saturated. Figure 6 also shows that the 0.6 center ratio is the highest throughput for a group of 80 users.

Figure 7 indicates that, for the initial distribution, the community of 80 cell users has been done. In the three systems up to 20 consumers, the performance is greater for the conventional FR system. The lowest performance for users is roughly $23 \mathrm{Mbps}$, irrespective of where they are in the FR unit, for 20 to 80 users. In comparison, the FFR1, with a linearly improvement in efficiency to 30 users, performs more than the FR, which stays practically the same for 30 to 80 users.

FFR2, on the other side, users from 10 to 40 linearly get the improved throughput values and it may go up to $36 \mathrm{Mbps}$ for users 40 and is almost constant for about 40 and 80 users regardless of where they are placed. In addition, the above remarks are clearly illustrated in Figure 7, in the fractional frequency reuse 2 (FFR2) schemes due to the equal 
distribution of resource blocks to the inner and outer cell regions that show the highest throughput. This mechanism ensures that applications from 40 applications and above have equal maximum performance values regardless of where they are. To make a description, Figure 7 indicates a clear distinction in cell mean throughput of all three forms of frequency reuse schemes, including conventional frequency reuse, FFR1 and FFR2. A significant observation is made here on the three distinct cell average scenarios. In terms of average throughput, in comparison with the two other frequencies reuse schemes, the FFR2 regime results in higher levels. The graph reveals that standard FR has a maximum speed of 23.80

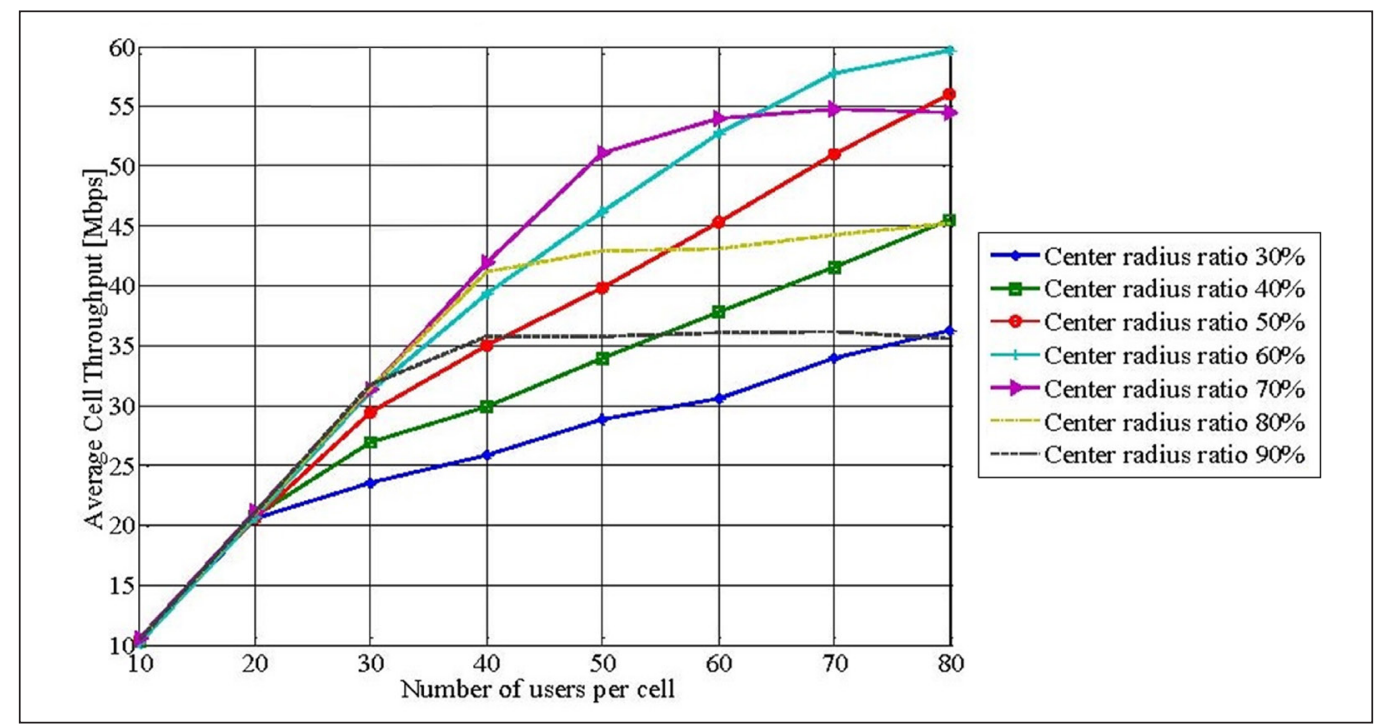

Figure 6. The average cell throughput at radius $750 \mathrm{~m}$ according to the number of users per cell to center radius ratio for FFR2

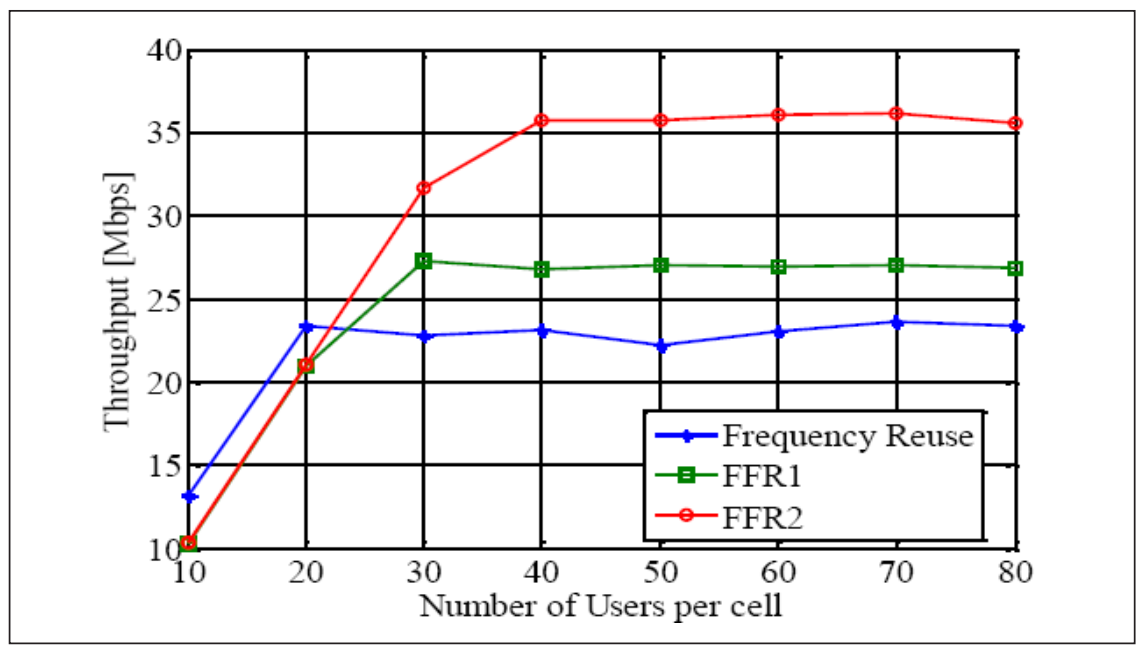

Figure 7. Comparison of FR, FFR1 and FFR2 scheme 
Table 4

The summary of maximum throughput (Mbps) for users and center to cell radius ratio $\left(C_{r r}\right)$ for both FFR1 and FFR2

\begin{tabular}{|c|c|c|}
\hline Users & FFR1 & FFR2 \\
\hline 80 & $48 \mathrm{Mbps}$ at $\mathrm{C}_{\mathrm{rr}} 0.5$ & 60 Mbps at $C_{\text {rr }} 0.6$ \\
\hline 70 & 45 Mbps at $C_{\mathrm{rr}} 0.5$ & 57.6 Mbps at $\mathrm{C}_{\mathrm{rr}} 0.6$ \\
\hline 60 & 44 Mbps at $C_{\mathrm{rr}} 0.6$ & $54.5 \mathrm{Mbps}$ at $\mathrm{C}_{\mathrm{rr}} 0.7$ \\
\hline 50 & 42 Mbps at $C_{r r} 0.6$ & $50 \mathrm{Mbps}$ at $\mathrm{Crr} 0.7$ \\
\hline 40 & 38 Mbps at $C_{\mathrm{rr}} 0.7$ & 42 Mbps at $C_{\text {rr }} 0.7$ \\
\hline 30 & 30 Mbps at $C_{\mathrm{rr}} 0.7$ & $32 \mathrm{Mbps}$ at $\mathrm{Crr} 0.8$ \\
\hline 20 & 20 Mbps at $C_{\mathrm{rr}} 0.7$ & $21 \mathrm{Mbps}$ at $\mathrm{C}_{\mathrm{rr}} 0.9$ \\
\hline 10 & 13 Mbps at $C_{\mathrm{rr}} 0.3$ & 11 bps at $\mathrm{C}_{\mathrm{rr}} 0.9$ \\
\hline
\end{tabular}

Mbps and FFR1 crosses 27.50 Mbps. FR reaches a cumulative flow value of $36.10 \mathrm{Mbps}$, on the other side. Table 4 summarizes the FFR1 and FFR2 potential performance along with a number of users and the cell radius node.

\section{CONCLUSION}

In this paper, the overall comparative evaluation on traditional Frequency Reuse (FR), FFR1 and FFR2 are presented. The general approach to avoid interference problem, the investigated mechanisms calculate the per-user SINR, capacity and throughput. It is found that the group of 80 users achieves the highest maximum throughput $48 \mathrm{Mbps}$ and $60 \mathrm{Mbps}$ for FFR1 and FFR2 at the cell radius ratio 0.5 and 0.6 , respectively. However, at center to cell radius ratio 0.5 , the maximum throughput is $55 \mathrm{Mbps}$ for the same user group. Therefore, it can be concluded that among the schemes, FFR2 provides the best throughput at 0.6 cell radius ratio for 80 users, which are considered as the optimal center to cell radius ratio and users, respectively.

\section{ACKNOWLEDGEMENT}

The authors would like to thank the Universiti Putra Malaysia special grant.

\section{REFERENCES}

Ali, S. H., \& Leung, V. C. (2009). Dynamic frequency allocation in fractional frequency reused OFDMA networks. IEEE Transactions on Wireless Communications, 8(8), 4286-4295. doi: 10.1109/ TWC.2009.081146

Bilios, D., Bouras, C., Kokkinos, V., Papazois, A., \& Tseliou, G. (2013). Selecting the optimal fractional frequency reuse scheme in long term evolution networks. Wireless Personal Communications, 71(4), 2693-2712. doi: https://doi.org/10.1007/s11277-012-0965-Z 
Chang, R. Y., Tao, Z., Zhang, J., \& Kuo, C. C. (2009, June 14-18). A graph approach to dynamic fractional frequency reuse (FFR) in multi-cell OFDMA networks. In 2009 IEEE International Conference on Communications (pp. 1-6). Dresden, Germany. doi: 10.1109/ICC.2009.5198612

Chung, S., Jang, S., \& Joe, I. (2014). Selective clustering scheme based on user equipment path and frequency reuse scheme for coordinated multi-point joint processing. IET Communications, 8(17), 2961-2970. doi: 10.1049/iet-com.2013.1186

Cong, L. S., Tuan, N. Q., \& Sandrasegaran, K. (2020). A general model of fractional frequency reuse: Modelling and performance analysis. VNU Journal of Science: Computer Science and Communication Engineering, 36(1), 38-45. doi: https://doi.org/10.25073/2588-1086/vnucsce.221

Elayoubi, S. E., Haddada, O. B., \& Fourestie, B. (2008). Performance evaluation of frequency planning schemes in OFDMA-based networks. IEEE Transactions on Wireless Communications, 7(5), 1623-1633. doi: 10.1109/TWC.2008.060458.

Group, I. W. (2004). IEEE standard for local and metropolitan area networks-part 16: Air interface for fixed broad-band wireless access systems. New Jersey, USA: Institute of Electrical and Electronics Engineers. doi: 10.1109/IEEESTD.2004.226664

Guo, T., \& Suárez, A. (2020). Fine-grained frequency reuse in centralized small cell networks. New Jersey, USA: IEEE Transactions on Mobile Computing. doi: 10.1109/TMC.2020.2981032

Hambebo, B. M., Carvalho, M. M., \& Ham, F. M. (2014, April 7-9). Performance evaluation of static frequency reuse techniques for OFDMA cellular networks. In Proceedings of the 11th IEEE International Conference on Networking, Sensing and Control (pp. 355-360). Miami, FL, USA. doi: 10.1109/ICNSC.2014.6819652

Hashima, S., Muta, O., Alghonimey, M., Shalaby, H., Frukawa, H., Elnoubi, S., \& Mahmoud, I. (2014, April 26-28). Area spectral efficiency performance comparison of downlink fractional frequency reuse schemes for MIMO heterogeneous networks. In 2014 International Conference on Information Science, Electronics and Electrical Engineering (Vol. 2, pp. 1005-1010). Sapporo, Japan. doi: 10.1109/InfoSEEE.2014.6947820

Hindia, M. N., Reza, A. W., \& Noordin, K. A. (2014). Investigation of a New Handover Approach in LTE and WIMAX. The Scientific World Journal, 2014, 1-11. doi: https://doi.org/10.1155/2014/246206

Kim, D. C., Kwon, Y. H., Kwak, J. S., Moon, S. H., \& Han, S. H. (2014). U.S. Patent No. 8,660,050. Washington, DC: U.S. Patent and Trademark Office.

Kim, Y. S., Choi, S. H., Jang, K. H., \& Hwang, H. S. (2010). U.S. Patent No. 7,821,976. Washington, DC: U.S. Patent and Trademark Office.

Lei, H., Zhang, L., Zhang, X., \& Yang, D. (2007, September 3-7). A novel multi-cell OFDMA system structure using fractional frequency reuse. In 2007 IEEE 18th International Symposium on Personal, Indoor and Mobile Radio Communications (pp. 1-5). Athens, Greece. doi: 10.1109/PIMRC.2007.4394228

Mannweiler, C., Sartori, C., Wegmann, B., Flinck, H., Maeder, A., Goerge, J., \& Winkelmann, R. (2020). Evolution of Mobile Communication Networks. In S. Mwanje \& C. Mannweiler (Eds.), Towards Cognitive Autonomous Networks: Network Management Automation for 5 G and Beyond (pp. 29-92). Croydon, UK: John Wiley \& Sons Ltd. doi: https://doi.org/10.1002/9781119586449.ch2 
Mao, X., Maaref, A., \& Teo, K. H. (2008, November 30 - December 4). Adaptive soft frequency reuse for inter-cell interference coordination in SC-FDMA based 3GPP LTE uplinks. In IEEE GLOBECOM 20082008 IEEE Global Telecommunications Conference (pp. 1-6). New Orleans, LO, USA. doi: 10.1109/ GLOCOM.2008.ECP.916

Nuaymi, L. (2007). WiMAX: technology for broadband wireless access. Chippenham, UK: John Wiley \& Sons.

Rumney, M. (2013). LTE and the evolution to $4 G$ wireless: Design and measurement challenges. Singapore: John Wiley \& Sons.

Saleh, A. M. M. (2020). Performance analysis of fractional frequency reuse schemes in downlink multi-relay multi-cell OFDMA and NOMA cellular networks (PhD Thesis). University of Calgary, Canada.

Sheu, T. L., Lin, B. J., \& Chou, Z. T. (2015). Analytical models for call blocking and dropping in sectorized cellular networks with fractional frequency reuse. Wireless Communications and Mobile Computing, 15(17), 2125-2140.

Simonsson, A. (2007, April 22-25). Frequency reuse and intercell interference co-ordination in E-UTRA. In 2007 IEEE 65th Vehicular Technology Conference-VTC2007-Spring (pp. 3091-3095). Dublin, Ireland. doi: 10.1109/VETECS.2007.633

Soultan, E. M., Nafea, H. B., \& Zaki, F. W. (2020). Interference Management for Different 5G Cellular Network Constructions. Wireless personal communications, 115(3) 1-20. doi: https://doi.org/10.1007/ s11277-020-07805-1

Stiakogiannakis, I. N., \& Kaklamani, D. I. (2010, September 26-30). Fractional frequency reuse techniques for multi-cellular WiMAX networks. In 21st Annual IEEE International Symposium on Personal, Indoor and Mobile Radio Communications (pp. 2432-2437). Instanbul, Turkey. doi: 10.1109/PIMRC.2010.5671728

Taranetz, M., Ikuno, J. C., \& Rupp, M. (2011, November 6-9). Capacity density optimization by fractional frequency partitioning. In 2011 Conference Record of the Forty Fifth Asilomar Conference on Signals, Systems and Computers (ASILOMAR). Pacific Grove, CA.

Wang, H. (2013). Biorthogonal frequency division multiple access cellular system with angle division reuse scheme. Wireless Personal Communications, 70(4), 1553-1573. doi: https://doi.org/10.1007/s11277012-0765-5

Wang, K., Li, H., Ma, J., Liu, P., \& Pan, H. (2014). Two-level scheme to maximise the number of guaranteed users in downlink femtocell networks. IET Communications, 8(16), 2917-2924. doi: 10.1049/ietcom.2013.0944

Zhang, H., Sun, Y., Lou, H. L., \& Chu, L. (2017). U.S. Patent No. 9,717,086. Washington, DC: U.S. Patent and Trademark Office.

Zhao, Y., Fang, X., Huang, R., \& Fang, Y. (2012). Joint interference coordination and load balancing for OFDMA multihop cellular networks. IEEE Transactions on Mobile Computing, 13(1), 89-101. doi: 10.1109/TMC.2012.224 
